\title{
(1]
}

'University of lowa, lowa City, IA, USA.

2Rady Children's Hospital, University of California, San Diego, CA, USA.

${ }^{3}$ Oakland University, William Beaumont School of Medicine, Rochester, MI, USA.

\section{Vocal cord dysfunction: a functional cause of respiratory distress}

Initially described as hysteria and then Munchausen's stridor, we now recognise vocal cord dysfunction as several disorders. Exercise-induced and spontaneously occurring phenotypes exist that benefit from different treatments. The former appears to respond to pharmacological pre-treatment with an anticholinergic aerosol; vocal cord training is the recommended treatment for the latter. The differential diagnosis includes consideration of neurological and anatomical abnormalities. Recognition and effective treatment is important to avoid misdiagnosis that results in unnecessary and ineffective medical care.

@ ERSpublications

VCD has several clinical and physiological phenotypes, which should be individually identified http://ow.ly/orfb309fMxh

\section{Background}

Dyspnoea is a complex psychophysiological sensation that occurs in a variety of cardiopulmonary and functional disorders. Increased work of breathing occurs when there is an increase in mechanical loading of the respiratory system. A physiological cause that increases mechanical loading and consequent dyspnoea occurs when the vocal cords adduct during inspiration when they should abduct to permit minimal obstruction to air flow. Functional vocal cord dysfunction can cause sudden onset of stridor and dyspnoea in the absence of identifiable neurological abnormality. Robley Dunglison, in 1842, described a disorder of the laryngeal muscles resulting in crowing inspiration and dyspnoea that he termed "hysteric croup" [1]. Episodic upper airway obstruction was subsequently termed "Munchausen's stridor" [2].
Vocal cord dysfunction (VCD) has been frequently misdiagnosed as asthma despite the difference between inspiratory stridor, the sound typical of upper airway obstruction, and the typical polyphonic expiratory wheeze of asthma [3-6].

\section{Demographics}

Our previously reported experience in a paediatric allergy and pulmonary specialty clinic involved a review of 49 sequential patients diagnosed with VCD [7]. Among those patients were two distinct phenotypes: $59 \%$ whose symptoms were limited to being exercise induced and 33\% whose VCD would occur spontaneously without identified stimuli; another $8 \%$ with spontaneously occurring VCD indicated that exercise would also cause symptoms. While asthma had been a common misdiagnosis for
Cite as: Weinberger $M$, Doshi D. Vocal cord dysfunction: a functional cause of respiratory distress. Breathe 2017; 13: 15-21. 
most, only 12 of the 49 had concomitant asthma in addition to VCD. The diagnosis of VCD was based, whenever possible, on direct laryngoscopy or reversible flattening of the inspiratory portion of the flow-volume loop during observed stridor. However, 17 of the 49, whose symptoms could not be observed, were diagnosed with VCD based on a convincing history of episodic inspiratory stridor that was rapid in onset and rapidly reversible.

At the time of diagnosis, the median age for those with exclusively exercise-induced VCD was 15 years (range $9-20$ years). The median age for those with spontaneously occurring VCD averaged 13.5 years (range $8-25$ years). The female/male ratio was high for both types of VCD, 1.6/1 in the patients with VCD only during exercise and 2.2/1 in the patients with spontaneously recurring VCD.

\section{Clinical presentation}

\section{Case 1}

A 15-year-old girl took a typical teenage summertime job in lowa, USA, called detasselling that involved working in the corn fields. (Detasseling is performed to cross-pollinate or hybridise two different varieties of corn. Fields of corn that will be detasselled are planted in alternate rows with two varieties of corn. By removing the pollenproducing tassels from all plants in one row, those plants will be fertilised by the other variety's pollen in the adjoining row. Detasselling work is typically performed by teenagers desiring to make some money during their summer vacation. For many in rural areas of the US Corn Belt, it essentially serves as a rite of passage as their first job.) She developed severe dyspnoea with respiratory sounds that were described as "wheezing" and was taken to the local emergency department where an injection of adrenaline relieved her symptoms. The next day, she again attempted detasselling with the same result. She then abandoned her goal of detasselling but continued for the next 3 weeks to have similar episodes of dyspnoea associated with respiratory sounds described by the patient, emergency department caregivers and her primary care paediatrician as "wheezing". However, when asked, the patient described that the "wheezing" was during inspiration only. These episodes would occur both spontaneously and with exertion, and would not respond to adrenaline as they did on the first 2 days. Trials by her primary care paediatrician of antiasthmatic medications, including inhaled albuterol, oral corticosteroids and inhaled corticosteroids, failed to either prevent or relieve acute symptoms when they occurred. Symptoms would last up to several hours, considerably limiting activity of this normally very active adolescent girl.

After 3 weeks of these daily symptoms, she was referred to our paediatric allergy and pulmonary clinic. Although initially asymptomatic, a treadmill

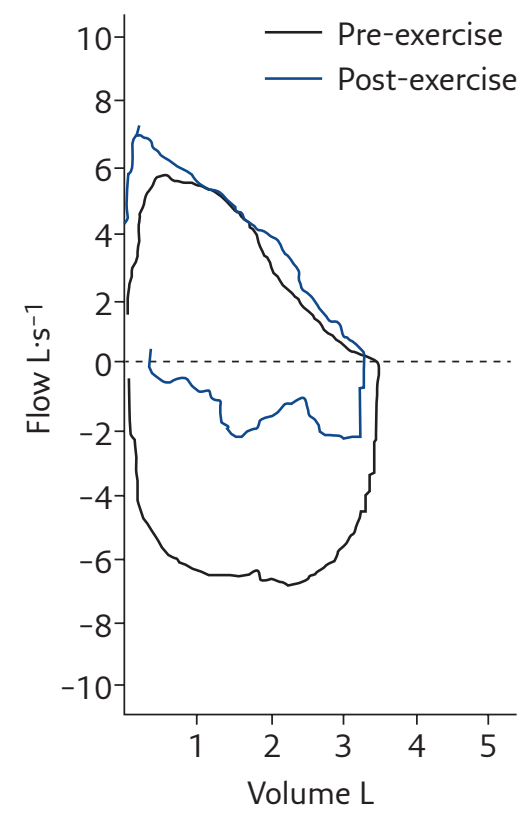

Figure 1 Spirometry before and after exercise in the girl described in Case 1 showing the marked decrease in the inspiratory portion of the flow-volume loop in association with dyspnoea and an inspiratory wheeze-like sound (technically a high-pitched stridor; see video 1 with audio in the supplementary material). Reproduced from [6] with permission from the publisher.

exercise test reproduced her dyspnoea and the inspiratory respiratory sound previously described as "wheezing". The sound was high-pitched and limited to inspiration. Spirometry before and after the onset of dyspnoea demonstrated inspiratory airway obstruction (figure 1). Flexible laryngoscopy during the episode demonstrated paradoxical vocal cord movement with adduction in inspiration and passive abduction on expiration (see video 1 in the supplementary material, with audio).

This demonstrated that her dyspnoea was from VCD. The evaluation included a large positive skinprick test to corn pollen. That explained the initial episodes as being consistent with an allergenspecific IgE mediated laryngeal oedema from intense exposure to the corn pollen during the detasselling procedure. (Corn pollen is relatively heavy and does not carry very far by wind. Exposure is generally occupational, rather than incidental as in the manner of light wind-carried pollens.) While adrenaline was effective for the episodes associated with the intense exposure to corn pollen, the subsequent episodes were the VCD.

Instructions by a speech pathologist provided this girl with the ability to stop the paradoxical movement when it would occur, but it continued to recur during vigorous activity interfering with her activities as a cheer leader. Suspecting a vagal mechanism for this, a trial of pre-exercise treatment with an anticholinergic inhaler, ipratropium (Atrovent oral inhaler; Boehringer Ingelheim, Ingelheim, Germany), reliably prevented the exercise-induced VCD [7]. This allowed her 
to resume her usual athletic activities, including cheerleading.

\section{Case 2}

A 15-year-old girl had a 1.5-year history of recurrent, extremely severe episodes of dyspnoea. These were sufficiently impressive to observers that paramedics were repeatedly called for urgent transportation to a local hospital emergency department. Symptoms would last for variable periods of time and occurred with sporadic frequency without any apparent inciting factors. She reported that exercise was tolerated without inducing the symptoms she described. Treatment with various antiasthmatic medications including inhaled and oral corticosteroids had provided no benefit. She had a history of multiple episodes where emergency intervention occurred and had been hospitalised several times where she received intravenous corticosteroids and vigorous use of inhaled bronchodilators, also without benefit.

When first seen at our paediatric allergy and pulmonary clinic, she was free of any symptoms of dyspnoea and her physical examination was normal. Initial spirometry was completely normal. Since the nature of her recurrent dyspnoea was not apparent from the history but appeared atypical for asthma, a bronchoprovocation with histamine was planned in order to assess whether asthma was the cause of her recurrent dyspnoea. While the patient watched the preparation of vials of the various concentrations of histamine needed for the progressive inhalational provocation, but before any had been administered, she began having severe respiratory distress with both inspiratory and expiratory wheezing-like sounds. Her spirometry changed from showing normal inspiratory and expiratory flow to severe obstruction in both phases of respiration (figure 2). Flexible laryngoscopy during the episode demonstrated virtually complete closure of the vocal cords, leaving only a small aperture for air movement with no abduction except briefly during speech (video 2 ).

Treatment focused on speech pathology to teach the patient control over her vocal cords when symptoms occurred. Treatment was only partially effective. She was able to control some episodes but not others. Lack of continuity for her care hampered progress. She and her single mother subsequently moved to Texas, and she was lost to follow-up.

\section{Clinical phenotypes}

Cases 1 and 2 illustrate the distinct phenotypes of VCD: exercise-induced and spontaneously occurring. Case 1 had components of both with paradoxical vocal cord movement; adduction on inspiration and normal abduction on expiration. Case 2 had no exercise-induced VCD but exhibited spontaneously occurring VCD with adduction during

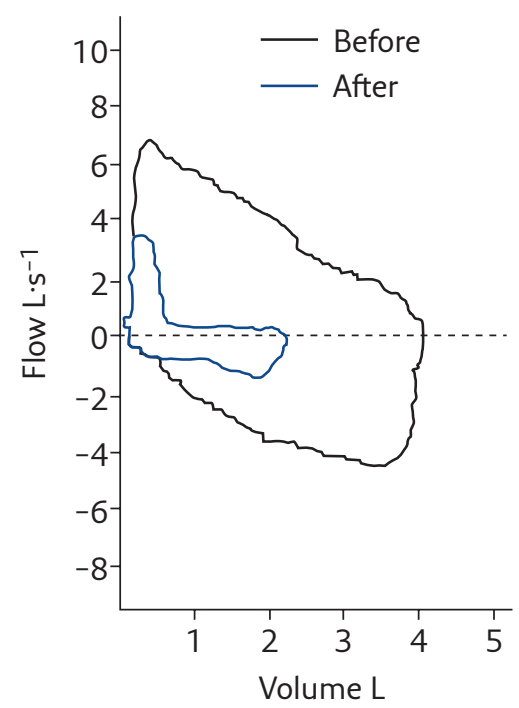

Figure 2 Spirometry before and after the onset of dyspnoea in the girl described in Case 2 showing the marked decrease in the inspiratory and expiratory portion of the flow-volume loop in association with an inspiratory wheeze-like sound (technically a high-pitched stridor on inspiration) and a monophonic wheeze on expiration (see video 2 with audio in the supplementary material). Reproduced from [6] with permission from the publisher.

both inspiration and expiration. NeWMAN et al. [3] described another phenotype, expiratory VCD. In their published series of VCD cases, $11 \%$ were reported to have only the expiratory adduction, and $31 \%$ had the combined inspiratory and expiratory adduction, while the remainder had the more typical paradoxical movement with adduction only on inspiration.

\section{Prevalence}

The prevalence of VCD is unknown because of both under- and overdiagnosis. The exerciseinduced VCD phenotype appears to be the more common phenotype. Typically, this is triggered by vigorous athletic activity and presents as dyspnoea on exertion associated with inspiratory stridor. The patient may complain of tightness in the throat and difficulty getting air in. RUNDELL and SPIERING [8] reported exercise-induced VCD in elite athletes, predominantly female, based solely on eliciting stridor during exercise, and listening over the larynx and chest to confirm their impression that the inspiratory sound they identified as stridor was heard in the laryngeal area. There was no endoscopic or spirometric evidence to confirm VCD, a deficiency acknowledged in their publication. Those authors claim that flattened or truncated inspiratory flowvolume loops to be insensitive in identifying VCD.

Contrary to the conclusions of RUNDELL and SPIERING [8], we have frequently heard inspiratory noise at maximal effort in well-conditioned and highly motivated athletes without evidence of upper airway obstruction, either by the flow-volume loop or direct visualisation with a flexible laryngoscope [9]. 
We suspect that very high air movement through the narrowest portion of a normal upper airway during maximal effort can result in a transient stridorous sound in the absence of any additional airway obstruction. We have never visualised VCD without having seen truncation of the inspiratory portion of the flow-volume loop where the FIF50/FEF50 (ratio of forced inspiratory flow to forced expiratory flow at $50 \%$ of vital capacity) was very low when symptomatic, rather than the usual ratio of about 1 present when the same patient was asymptomatic. Therefore, the assumption that increased inspiratory laryngeal noise during exercise as the sole finding is insufficient to confirm the diagnosis of VCD.

Less common but associated with the more severe pattern of upper airway obstruction as illustrated in Case 2 (video 2 ) are those patients who experience adduction of the vocal cords on both inspiration and expiration, often also associated with adduction of the false vocal cords $[10,11]$. This produces an inspiratory and expiratory wheezinglike sound, chest tightness and shortness of breath. The acute onset and severity of symptoms in some patients with this pattern of VCD has resulted in emergency intervention with endotracheal intubation or tracheotomy for severe upper airway obstruction [11].

\section{Differential diagnosis}

There are other causes of upper airway obstruction that may be confused with VCD. They include virtually any cause of acquired stridor. A particularly relevant consideration for exercise-induced stridor is exercise-induced laryngomalacia [12-16]. VCD from paradoxical movement (figure 1 and video 1) and exercise-induced laryngomalacia both cause exercise-induced dyspnoea due to upper airway obstruction on inspiration. Spirometry will result in virtually identical flattening of the inspiratory portion of the flow-volume loop. However, the effect for VCD occurs from paradoxical vocal cord movement, while collapse of the laryngeal structures is the cause of the upper airway obstruction with laryngomalacia. In at least some cases, this appears to be related to the previous presence of infantile laryngomalacia [17].

VCD has also been seen with Chiari 1 malformations. In a review of 31 young children with Chiari 1 malformations, VCD was seen in $26 \%$, all under the age of 3 years [18]. Case 3 illustrates such a patient seen by us.

\section{Case 3}

A 17-month-old child had harsh nocturnal coughing and occasional stridor. She was initially treated as having asthma, without response. Intermittent paradoxical vocal cord moment was seen during flexible bronchoscopy. The age was so atypical for functional VCD that head magnetic resonance

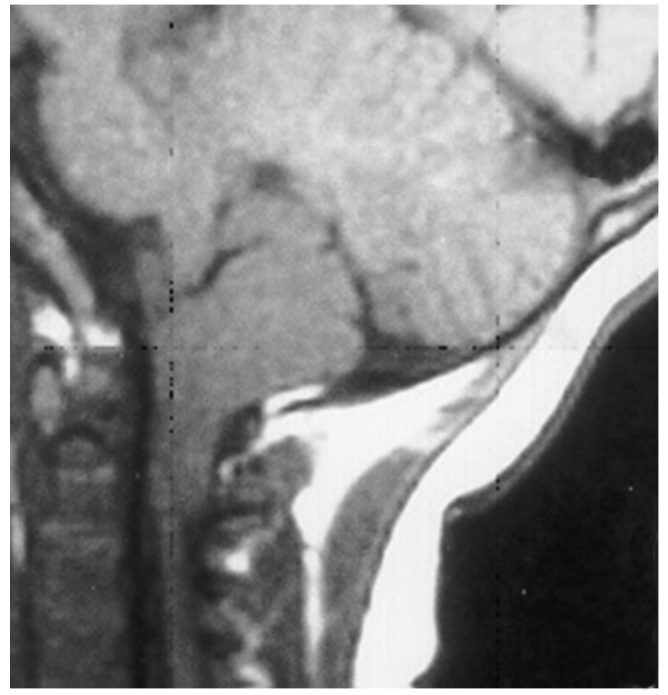

Figure 3 Magnetic resonance image demonstrating herniation of the cerebellar tonsil below the foramen magnum consistent with a Chiari 1 malformation.

imaging was performed to examine for a Chiari 1 malformation. When that was confirmed (figure 3), decompressive neurosurgery eventually eliminated the respiratory symptoms.

Other disorders that can be confused with VCD included vocal cord paresis or paralysis [19]. Subglottic stenosis may also present in a manner that can initially be suggestive for vocal cord dysfunction, as illustrated by the following Case 4 .

\section{Case 4}

A 14-year-old girl was seen for a history of recurrent respiratory distress associated with stridor. VCD was suspected from the history. Since she was asymptomatic when first seen and had normalappearing spirometry, other than a mild decrease in the inspiratory flow rate, she was advised to return the next time she had similar symptoms. When seen several weeks later with an acute episode of dyspnoea and stridor, there was marked flattening of the inspiratory portion of her flow-volume loop. A flexible fibreoptic laryngoscopy visualised completely normal vocal cord movement. Flexible bronchoscopy then demonstrated profound subglottic stenosis. The 3.5-mm diameter bronchoscope completely obstructed the airway of this normal-sized 15 -year-old girl just below the vocal cords. She was subsequently diagnosed with relapsing polychondritis similar to a previous report [20].

Individual situations may be confounded by the presence of more than one disorder. The presence of asthma complicated by the presence of VCD has both previously reported and seen by us [21].

\section{Case 5}

A 14-year-old girl with athletic ambitions as a sprinter was seen for episodes of spontaneous 
dyspnoea and dyspnoea on exertion. She was an exceptional student academically in addition to being a competitive sprinter. She had a long history of asthma and severe seasonal allergic rhinoconjunctivitis. Evaluation confirmed impressive allergy skin tests to pollens. A carefully taken history revealed symptoms of light-headedness and tingling in her hands and feet associated with the periods of spontaneous dyspnoea consistent with hyperventilation. A treadmill exercise stress test demonstrated both exercise-induced bronchospasm and transient upper airway obstruction [9]. Diagnoses of exercise-induced asthma, exerciseinduced VCD and hyperventilation attacks were added to her diagnoses of chronic asthma and pollen allergy. Counselling resulted in cessation of the hyperventilation episodes; albuterol and ipratropium metered-dose inhaler (MDI) use prior to exercise enabled her to compete effectively without the previous post exercise dyspnoea associated with both stridor and VCD. Usual medications for asthma and allergic rhinoconjunctivitis improved those symptoms with further control provided by immunotherapy from pollen-extract injections. She demonstrated impressive understanding of her multiple disorders and their treatment.

\section{Pathophysiology of VCD}

Several studies have suggested that a spontaneous onset of VCD is often associated with underlying psychiatric disorders. Psychiatric consultation for further therapy and consideration of underlying somatoform disorders has been recommended [22]. In a report by SELNER et al. [23], three patients were described with spontaneous onset of VCD symptoms attributed to psychological factors. A study by Gavin et al. [24] described 12 patients with VCD occurring only at times of anxiety with no relationship to activity or exercise. There is a report suggesting an association of VCD with sexual abuse [25]. NEWMAN et al. [3] reported a previous psychiatric diagnosis in $73 \%$ of patients with VCD. A higher incidence of VCD has been reported in female patients with an underlying psychological condition [26]. Stress and emotions, as well as times of increased panic or anxiety, have been suggested as triggers for VCD [27]. VCD was also reported to be associated with social stressors in some paediatric patients involved in organised sports [28].

In adults, but not in children, chronic cough has been described in association with abnormal vocal cord movement [27].

\section{Treatment of VCD}

We have observed that an anticholinergic aerosol administered prior to exercise appears to prevent exercise-induced VCD. Support for vagal mediation in exercise-induced VCD is suggested by reports that vagal nerve stimulators, used for patients with intractable seizures, cause VCD as a complication [29, 30]. Laryngopharyngeal dysfunction, coughing and voice changes were reported in these patients. Moreover, an altered autonomic balance has been suggested as a cause of VCD, since true and false vocal cords derive motor innervation from the vagus nerve [31]. Because an apparent vagally mediated reflex was involved in VCD, we speculated that an anticholinergic aerosol might prevent exercise-induced VCD afferent and/or efferent vagal innervation in the pharynx. An ipratropium bromide aerosol (Atrovent MDI) was prescribed and used prior to exercise in six of our patients initially described with exercise-induced VCD [7] who all reported prevention of exercise-induced symptoms. Use of other inhalers, such as albuterol, prior to exercise in four of those six previously diagnosed with asthma had been ineffective, which argues against a placebo effect. We have since utilised the anticholinergic aerosol for many more patients and have continued to see apparent effectiveness as prophylaxis when used prior to exercise but not as a means of reversing symptoms after they occur. A placebo-controlled clinical trial is needed to confirm these repeated clinical observations. One of the authors (D. Doshi) has used once-daily tiotropium, a long-acting anticholinergic aerosol, as a daily preventative for patients experiencing frequent spontaneous VCD, with apparent positive effect.

A study by Sullivan et al. [32] described teaching 20 female athletes "coordinated thoracicabdominal breathing exercises" when symptoms of VCD occurred during exercise. 19 of the 20 indicated being able to control their symptoms after 6 months. However, it was not clear whether complete resolution of the problem had occurred in any. In our patients with exercise-induced VCD, symptoms generally subsided with a decrease or cessation of exercise. Breathing exercises such as those described by SuLLIVAN et al. [32] appeared, to us, difficult to apply during active athletic activity. Our continued successful prevention of exerciseinduced VCD with an inhaled anticholinergic aerosol is efficient, benign and more realistically applicable than attempting breathing exercises during peak aerobic activity.

Speech therapy, relaxation, biofeedback, hypnosis and breathing techniques are interventions for VCD that have been described previously [3336]. Christopher et al. [21] described improvement in symptoms 3-21 months after speech therapy in five patients. In our published report, eight out of 11 contacted patients with spontaneously occurring VCD followed our recommendations for speech therapy [7]. Although the patients reported that the techniques taught by our speech therapist enabled them to control symptoms when they occurred, recurrences continued for up to 12 months. Of three who did not follow our recommendation for speech therapy, recurrent symptoms persisted for 4,12 and 60 months, respectively [7]. 
ANBAR and HeHIR [36] used hypnosis to assist in confirming the diagnosis by inducing spontaneously occurring severe VCD, which was then observed with flexible laryngoscopy. They then utilised hypnosis to have the patient "relax his neck" with conversion of the vocal cord movement from previous adduction to normal abduction during inspiration.

\section{Natural history of VCD}

The median duration of symptoms prior to our evaluation was 12 months (range 3 weeks to 7 years) and 6 months ( 1 week to 5 years) prior to our diagnosis for exercise-induced and spontaneously occurring VCD, respectively, irrespective of intervention [7]. We were able to contact 28 out of 49 patients included in our initial report a median time of 3 years (range 0.5-12 years) after the last of the clinic visits to question them about long-term outcome. The median time until resolution of symptoms was 5 and 4 months for those with exercise-induced and spontaneous VCD, respectively, but there was a great deal of variability ranging from 1 week to
5 years, irrespective of intervention. However, six patients with exercise-induced VCD for whom the ipratropium MDI had not been prescribed indicated when contacted that they had quit the competitive athletic activity that had been associated with their symptoms [7].

\section{Conclusion}

VCD has several clinical and physiological phenotypes, which should be individually identified. Treatment should relate to those variations. Exercise-induced VCD appears to be readily prevented by pre-treatment with an anticholinergic aerosol, although those clinical impressions need to be confirmed with a controlled clinical trial. Spontaneously occurring VCD may benefit from training by a speech pathologist to learn effective voluntary control over the vocal cords. It is critical to consider the differential diagnosis of vocal cord disorders which can include abnormalities of the larynx causing exercise induced laryngomalacia and neurological abnormalities such as the Chiari 1 malformation.

Supplementary material

This article has supplementary material available from breathe.ersjournals.com

\section{Conflict of interest}

None declared.

\section{References}

1. Dunglison R. Practice of Medicine; or a treatise on specia pathology and therapeutics. Phladelphia, Lea \& Blanchard, 1842

2. Patterson R, Schatz M, Horton M. Munchausen's stridor: non-organic laryngeal obstruction. Clin Allergy 1974; 4 307-310

3. Newman KB, Mason UG 3rd, Schmaling KB. Clinical features of vocal cord dysfunction. Am J Respir Crit Care Med 1995; 152 1382-1386

4. McFadden ER Jr, Zawadski DK. Vocal cord dysfunction masquerading as exercise-induced asthma. a physiologic cause for "choking" during athletic activities. Am J Respir Crit Care Med 1996; 153: 942-947.

5. Thomas PS, Geddes DM, Barnes PJ. Pseudo-steroid resistant asthma. Thorax 1999; 54: 352-356.

6. Weinberger M, Abu-Hasan M. Pseudo-asthma: when cough, wheezing, and dyspnea are not asthma. Pediatrics 2007; 120 855-864.

7. Doshi D, Weinberger M. Long-term outcome of vocal cord dysfunction. Ann Allergy Asthma Immunol 2006; 96 : 794-799.

8. Rundell KW, Spiering BA. Inspiratory stridor in elite athletes Chest 2003; 123: 468-474.

9. Abu-Haan M, Tannous B, Weinberger M. Exercise-induced dyspnea in children and adolescents. If not asthma, then what? Ann Allergy Asthma Immunol 2005; 94: 366-371.

10. Wood RP, Milgrom H. Vocal cord dysfunction. J Allergy Clin Immunol 1996; 98: 481-485.
11. Goldstein R, Bright J, Jones SM, et al. Severe vocal cord dysfunction resistant to all current therapeutic interventions. Respir Med 2007; 1-1: 857-858.

12. Smith RJ, Bauman NM, Bent JP, et al. Exercise-induced laryngomalacia. Ann Oto Rhinol Laryngol 1995; 104: 537-541.

13. Bent JP, Miller DA, Kim JW, et al. Pediatric exerciseinduced laryngomalacia. Ann Oto Rhinol Laryngol 1996; 105: 169-175.

14. Björnsdóttir US, Gudmundsson K, Hjartarson $H$, et al. Exercise-induced laryngochalasia: an imitator of exerciseinduced bronchospasm. Ann Allergy Asthma Immunol 2000; 85: 387-391.

15. Manell DL, Arjmand EM. Laryngomalacia induced by exercise in a pediatric patient. Int J Ped Otorhinolaryngol 2003 67: 999-1003

16. Christensen $\mathrm{P}$, Thomsen SF, Rasmussen N, et al. Exerciseinduced laryngeal obstructions objectively assessed using EILOMEA. Eur Arch Otorhinolaryngol 2010; 267: 401-407.

17. Hilland M, Røksund OD, Sandvik L, et al. Congenital laryngomalacia is related to exercise induced laryngeal obstruction in adolescence. Arch Dis Child 2016; 101: 443-448.

18. Greenlee JDW, Donovan KA, Hasan DM, et al. Chiari I malformation in the very young child: the spectrum of presentations and experience in 31 children under age 6 years. Pediatrics 2002; 110: 1212-1219.

19. Morris MJ, Christopher KL. Diagnostic criteria for the classification of vocal cord dysfunction. Chest 2010; 138: 1213-1223. 
20. Kim C-M, Kim B-S, Cho K-J, et al. Laryngotracheal involvement of relapsing polychondritis in a Korean girl. Pediatr Pulmonol 2003; 35: 314-317.

21. Christopher KL, Wood RP 2nd, Eckert RC, et al. Vocal-cord dysfunction presenting as asthma. N Engl J Med 1983; 308: 1566-1570.

22. Fritz GK, Fritsch S, Hagino O. Somatoform disorders in children and adolescents: a review of the past 10 years. J Am Acad Child Adolesc Psychiatry 1997; 36: 1329-1338.

23. Selner JC, Staudenmayer H, Koepke JW, et al. Vocal cord dysfunction: the importance of psychologic factors and provocation challenge testing. J Allergy Clin Immunol 1987; 79: 726-733.

24. Gavin LA, Wamboldt M, Brugman S, et al. Psychological and family characteristics of adolescents with vocal cord dysfunction. J Asthma 1998; 35: 409-417.

25. Freedman MR, Rosenberg SJ, Schmaling KB. Childhood sexual abuse in patients with paradoxical vocal cord dysfunction. J Nerv Ment Dis 1991; 179: 295-298.

26. Leo RJ, Konakanchi R. Psychogenic respiratory distress: a case of paradoxical vocal cord dysfunction and literature review. Prim Care Companion J Clin Psychiatry 1999; 1: 39-46.

27. Murry T, Tabaee A, Aviv JE. Respiratory retraining of refractory cough and laryngopharyngeal reflux in patients with paradoxical vocal fold movement disorder. Laryngoscope 2004; 114: 1341-1345

28. Powell DM, Karanfilov BI, Beechler KB, et al. Paradoxical vocal cord dysfunction in juveniles. Arch Otolaryngol Head Neck Surg 2000; 126: 29-34.

29. Vassilyadi M, Strawsburg RH. Delayed onset of vocal cord paralysis after explantation of a vagus nerve stimulator in a child. Childs Nerv Syst 2003; 19: 261-263.

30. Zalvan C, Sulica L, Wolf S, et al. Laryngopharyngeal dysfunction from the implant vagal nerve stimulator. Laryngoscope 2003; 113: 221-225.

31. Ayres JG, Gabbott. Vocal cord dysfunction and laryngeal hyperresponsiveness: a function of altered autonomic balance? Thorax 2002; 57: 284-285.

32. Sullivan MD, Heywood BM, Beukelman DR. A treatment for vocal cord dysfunction in female athletes: an outcome study. Laryngoscope 2001; 111: 1751-1755.

33. Earles J, Kerr B, Kellar M. Psychophysiologic treatment of vocal cord dysfunction. Ann Allergy Asthma Immunol 2003; 90: 669-671.

34. Miller S. Voice therapy for vocal fold paralysis. Otolaryngol Clin North Am 2004; 37: 105-119.

35. Sandage M, Zelazny S. Paradoxical vocal fold motion in children and adolescents. Lang Speech Hear Serv Sch 2004; 35: 353-362.

36. Anbar RD, Hehir DA. Hypnosis as a diagnostic modality for vocal cord dysfunction. Pediatrics 2000; 106: e81. 